\title{
Application of polarized IR / NIR spectroscopic imaging for analysis of polymer products
}

\author{
by Y. Hikima*, T. Nakamura*, Y. Taya*, and M. Ohshima*
}

${ }^{*}$ Kyoto University, Department of Chemical Engineering, A4-021, Katsura Campus, 615-8510, Kyoto, JAPAN, hikima@cheme.kyoto-u.ac.jp

\begin{abstract}
IR / NIR spectroscopic imaging technique is powerful tool to visualize the chemical distribution in objects. In addition, the combination of linear polarized light and IR / NIR spectroscopic imaging technique enables to visualize simultaneously the distributions of anisotropy and chemical composition in the materials. In this study, polarized IR / NIR spectroscopic imaging techniques were applied for the analysis of polymer products. NIR spectroscopic birefringence imaging technique was developed and applied for analyzing an injection molded polymer product. Two linear polarizers were inserted in the optical system of a push-bloom type NIR spectroscopic imaging system. The obtained NIR spectra changed depending on not only the chemical structure but also the birefringence of sample. The influence of birefringence on NIR spectrum was extracted by data post-processing, and the magnitude and direction of birefringence were calculated by curve fitting. By using proposed technique, it was succeeded to visualize the thickness distribution calculated using near-infrared absorption, and the birefringence image which reflected the flow history during injection molding.
\end{abstract}

\section{Introduction}

Infrared (IR) and near-infrared (NIR) spectroscopy have been widely used as the analytical method for chemical structure of various materials. Combining IR camera and the spectrometer enables the hyperspectral imaging in IR and NIR regions. IR/NIR spectroscopic imaging provides lots of images taken with various wavelengths, namely, the hyperspectral cube. This high-dimensional data contains IR/NIR spectra at each position. By calculating the chemical composition, the reaction ratio, crystallinity etc. from each spectrum, we can obtain the chemical image of the sample [1].

The polarization measurement techniques are commonly used for investigating the anisotropy in various polymeric materials. For example, the polarized IR spectroscopy is applied for evaluating the molecular orientation in polymers [2] using the polarization dependence of infrared absorption, namely, infrared dichroism. On the other hand, it is easy to detect the birefringence, which is the anisotropy of refractive index, of a transparent anisotropic material by sandwiching the sample between two linear polarizers. The polarized optical microscope or birefringence imaging techniques are commonly applied for the morphological analysis in polymers or the biological tissues [3].

We have developed the advanced imaging technique for polymer products by combining the polarization analysis with IR/NIR spectroscopic imaging techniques. In this paper, two applications are reported: the polarized IR spectroscopic imaging of a polymer composite, and NIR birefringence imaging of injection molded polymer products.

\section{Polarized Infrared spectroscopic imaging}

\subsection{Measurement}

The measurement of transmission infrared spectroscopic imaging was conducted with Spotlight 400 (PerkinElmer). An infrared polarizer (KRS-5, wire-grid) was inserted between the sample stage and the array detector. The spatial resolution was $6.25 \mu \mathrm{m}$, and the spectral resolution was $4 \mathrm{~cm}^{-1}$. The accumulation number was 8 . The IR spectroscopic imaging was conducted with different polarizer angles from 0, 45, 90 and 135 degrees.

\subsection{Analysis}

From polarized IR spectra measured with four different polarizer angles, two characteristic values of molecular orientation: the in-plane orientation function and the orientation azimuth angle, can be calculated using four-polarization algorithm [3]. The former indicates the magnitude of molecular orientation and the latter indicates the direction of molecular orientation in the sample plane.

\section{Polarized Infrared spectroscopic imaging}

\subsection{Measurement}

The transmission NIR spectroscopic imaging was conducted with a push-bloom type NIR hyperspectral imaging system (Compovision, Sumitomo Electric Industries) which is composed of a light source, a movable sample stage, and 
NIR hyperspectral camera. One of the NIR polarizers (POLAX-30IR, Luceo) was inserted between the sample stage and the detector, and the other one was inserted between the sample stage and the light source. The spectral resolution was $6.3 \mathrm{~nm}$.

\subsection{Analysis}

NIR spectroscopic images were measured with three different optical systems: the single polarizer system, the crossed polarizers system, and the parallel polarizers system. The NIR spectra measured with two polarizers were described as trigonometric functions with absorption peaks. From the obtained spectra at each pixel, the effect of birefringence on the NIR transmission spectrum was extracted, and the retardation, which is the product of the birefringence and the sample thickness, was calculated.

\section{Results and Discussion}

\subsection{Polarized Infrared spectroscopic imaging of a polymer composite}

A film of the polypropylene (PP) / hydrophobic-modified cellulose nanofiber (HM-CNF) composite was fabricated at $190^{\circ} \mathrm{C}$ using a hot-pressing machine. The film thickness was $11 \mu \mathrm{m}$. The morphology in this film was investigated using polarized IR spectroscopic imaging method. Fig. 1 (a) shows the polarized optical microscopic image of a PP/HM-CNF composite. A large PP spherulite and a bundle of CNFs can be clearly visualized. However, it is troublesome at the other positions to distinguish which chemical component is oriented. Here, the polarized FTIR imaging technique was employed. The in-plane orientation function was calculated from a spectral band at $840 \mathrm{~cm}^{-1}$ (PP, crystal) using four polarization method [2]. Fig. 1(b) shows the FTIR image of in-plane orientation function at $840 \mathrm{~cm}^{-1}$ in the PP/HM-CNF composite. This image visualized only the morphology of PP crystal. Thus, this technique is useful to investigate the orientation behaviour in the multi-component system.

\subsection{NIR birefringence imaging of an injection molded polymer product}

A disc-shaped polystyrene (PS) plate was molded using an injection molding machine. This one-piece plate was composed of an inner disc part (thickness: $1.0 \mathrm{~mm}$ ) and an outer peripheral part (thickness: $1.5 \mathrm{~mm}$ ). During the injection molding process, the molten PS was injected at the center of the mold cavity and flowed in the radial direction. NIR birefringence imaging was applied for evaluating the flow history in this PS product. Fig. 2 (a) shows the NIR image of absorbance at $1148 \mathrm{~nm}$ obtained with single polarizer system. The absorbance increased at the outer peripheral part obeying Lambert-Beer's law. Thus, the absorbance image reflects the thickness distribution in the sample. Fig. 2(b) shows the NIR image of retardation of the injection molded PS plate. The large retardation was observed around the center of plate because the flow rate of molten PS was large during injection molding. The retardation gradually decreased along the radial direction.

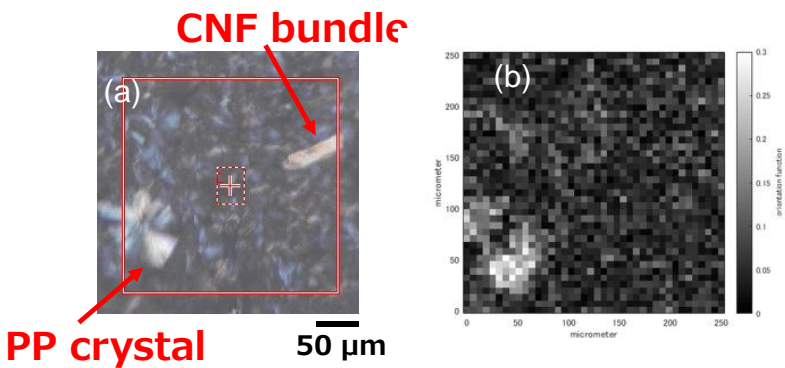

Fig. 1 (a) Polarized optical image and (b) FTIR image of in-plane orientation function at $840 \mathrm{~cm}^{-1}(P P)$ of $P P / H M$ CNFs composite
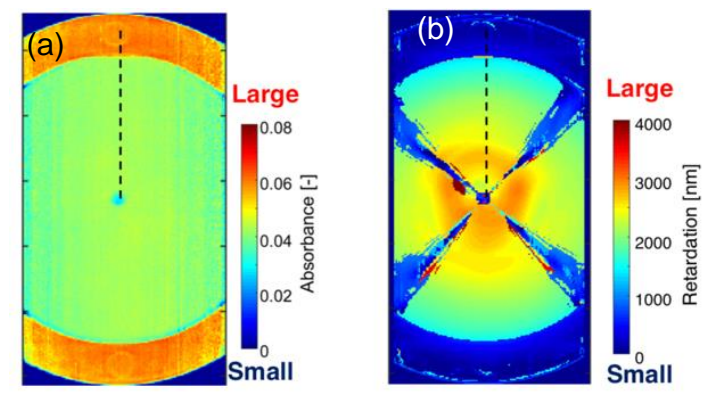

Fig. 2 NIR images of (a) absorbance at $1148 \mathrm{~nm}$ and (b) retardation of an injection molded PS plate

\section{REFERENCES}

[1] S. Muroga, Y. Hikima, M. Ohshima, Near-Infrared Spectroscopic Evaluation of the Water Content of Molded Polylactide under the Effect of Crystallization., Appl Spectrosc. 2017;71:1300-1309.

[2] Y. Hikima, J. Morikawa, T. Hashimoto, FT-IR Image Processing Algorithms for In-Plane Orientation Function and Azimuth Angle of Uniaxially Drawn Polyethylene Composite Film, Macromolecules. 2011; 44: 3950-3957.

[3] M. Shribak, R. Oldenbourg, Techniques for fast and sensitive measurements of two-dimensional birefringence distributions, Applied Optics. 2003;42:3009-3017. 